\title{
Tierexperimentelle Untersuchung über die Wirkung von Solcoseryl auf das Knochenwachstum bei
} Kieferfrakturen (1)

\author{
Jinichi Fukuda \\ First Department of Oral Surgery (Director : Prof. Nagayosh Yamada) \\ Kyushu Dental College, Kitakyushu, Japan \\ Received on 14th August, 1983.
}

\section{Einleitung}

In Japan wird Solcoseryl in Injektionsform zur Behandlung des Magenulcus ${ }^{1,2)}$, von Carcinompatienten ${ }^{3}{ }^{4}$ ) und bei zerebralen Durchblutungsstörung ${ }^{5}{ }^{6}$ ) eingesetzt. Knochenfrakturen und -Defekt wurden bisher mit Solcoseryl nicht behandelt.

Ziel dieser orientierenden Studie ist es zu prüfen, ob Solcoseryl die Heilung von Knochenfrakturen qualitativ und quantitativ beeinflusst.

\section{Material und Methode}

Operationstechnik

Es wurde das folgende Tiermodell gewählt: Die Tiere wurden mit Pentobarbitalum Sodium (0.4 ml/kg, i.v.) anästhesiert. Der Unterkiefer wird auf der rechten Seite durch einen Hautschnitt freigelegt und das Weichgewebe sowie das Periost bis zum Alveolarkamm abgeklappt. Zwischen dem 3. Prämolaren und dem 1. Molaren wird die Corticalis bis zur Spongiosa mit einer Lindemannfräse durchtrennt. Anschliessend wird der Kiefer mit dem Gnathotom gebrochen. Vor dem Durchtrennen des Kiefers werden eine AO-Platte (4-DCP) nach Prof. Spiessl ${ }^{7}$ ) an die Kieferform angepasst und die Schraubenlöcher vorgebohrt. Damit lassen sich die Kieferbruchstücke in ihrer ursprünglichen Lage stabil fixieren. Es entsteht ein Spalt in der Corticalis von ca. $2 \mathrm{~mm}$ (Abb. 1). Nach dem Reponieren wird das Periost über die Platte zrückgeklappt und mit Catgut vernäht. Anschliessend werden Muskeln und Haut mit Silk vernäht und das Nahtmaterial nach 1 Woche entfernt. Jeder Hund erhielt von 1. -7 . Tag p.o. AB-PC i.m.

Tiere

6 japanische Mischlinge-Hunde von durchschnittlich $10 \mathrm{Kg}$ Gewicht, die unter gleichen Bedingungen bei gekochtes Fleisch, Reis und Gemüse gehalten waren, wurden in zwei Gruppen zu 3 Tieren eingeteilt (Tabelle 1).

Gruppe A dients als Kontrolle und blieb ohne Behandlung. Die Tiere der Gruppe B erhielten täglich $2 \mathrm{ml} / \mathrm{Kg}$ Solcoseryl i.v. (In diesem Fall wurde es Solcoseryl von TOBISHI PHARMACEUTICAL Co., LTD. angeboten.) 


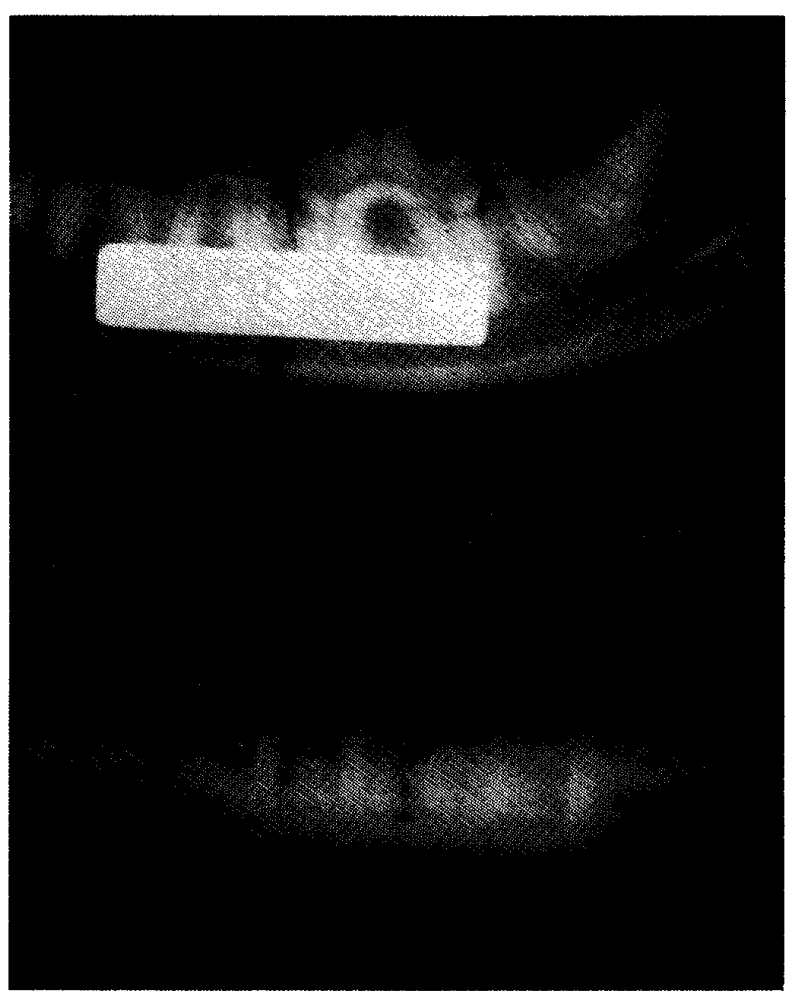

Abb. 1 Röntgenbild nach der Operation
Tabelle 1

\begin{tabular}{c|c|c|c|c}
\hline \hline Hund Nr. & Gewicht Kg & Sex & \multicolumn{2}{|c}{ Gruppe } \\
\hline 1 & 10 & $\mathrm{f}$ & Kontrolle & \\
2 & 10 & $\mathrm{~m}$ & Kontrolle & $\mathrm{A}$ \\
3 & 7 & $\mathrm{~m}$ & Kontrolle & \\
\hline 4 & 12 & $\mathrm{~m}$ & Solcoseryl & \\
5 & 13.5 & $\mathrm{~m}$ & Solcoseryl & $\mathrm{B}$ \\
6 & 10 & $\mathrm{~m}$ & Solcoseryl & \\
\hline
\end{tabular}

Röntgen und Histologie

Von den Tieren wurden nach 1., 2 . und 3. Woche getötet. Die Fraktur wurde nach der Opferung des Hundes und Präparation des Kiefers von apikal und lateral geröntgt. Des präparierten Kiefers wurden in $10 \%$ neutrales Formalin fixiert. Entkalkung mit $5 \%$ Salpetersäure. Einbettung in Celloidin. Schnittdicke $15 \mu \mathrm{m}$. Färbung mit Hämatoxylin-Eosin.

\section{Ergebnisse}

Die histologischen Befunde sind in den Abbildungen 2-13 dargestellt. Die Schnitte wurden bei 4 und 10 facher Vergrösserung mit Mikroskop fotografiert.

1. Woche nach der Operation

Kontrolle (Abb. 2,3)

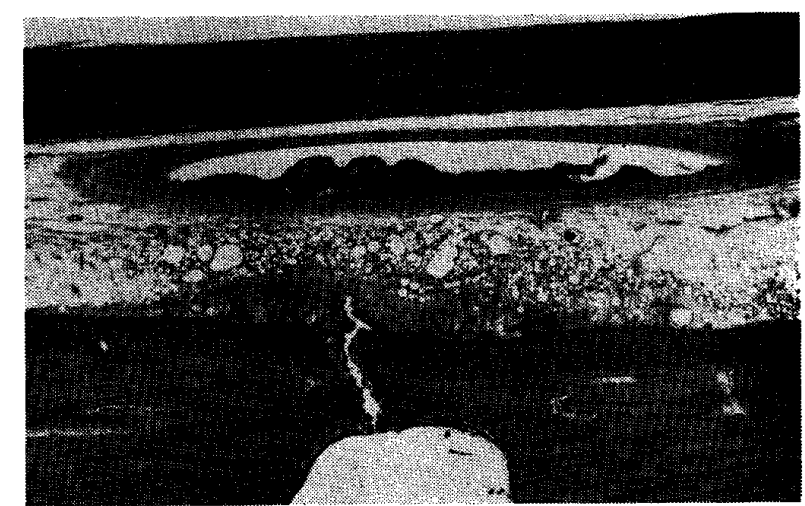

Abb. 2 Histologische Bild im Canalis mandibulae nach der 1 Woche. (Kontrolle)

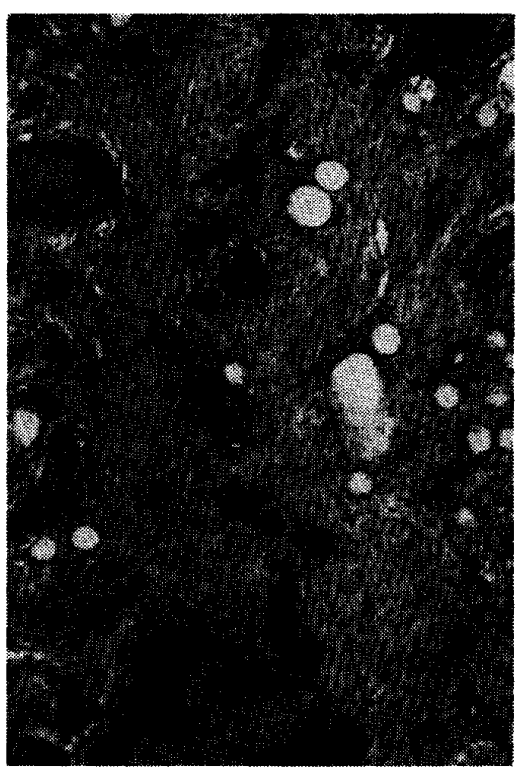

Abb. 3 Histologische Bild in der Spongiosa nach der 1 Woche. (Kontrolle) 
Es besteht geringe Callusbildung an der Corticalis und entzündliche Reaktionen in der Spongiosa. Es findet sich lockere, vereinzelte Neubildung des Bindegewebe und einige Kapillaren in der Spongiosa.

Solcoseryl (Abb. 4,5)

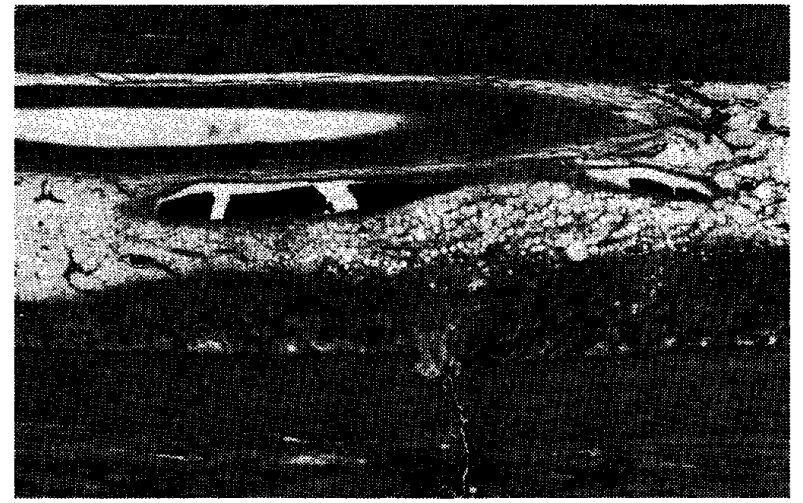

Abb. 4 Histologische Bild im Canalis mandibulae nach der 1 Woche. (Solcoseryl)

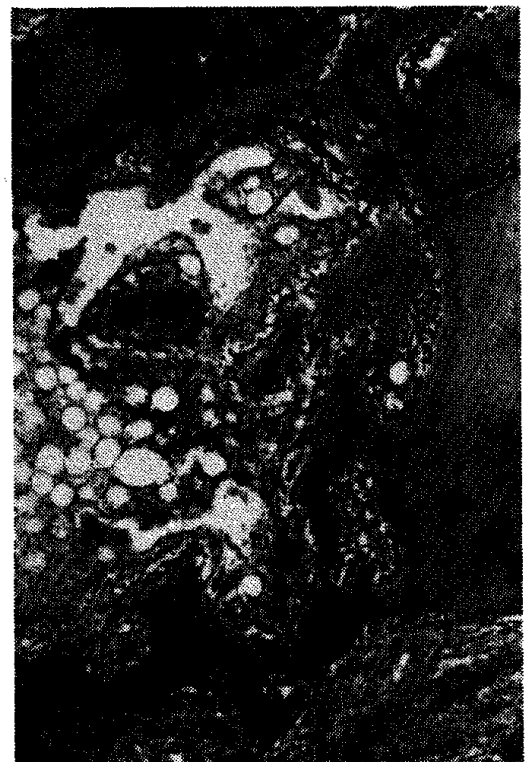

Abb. 5 Histologische Bild in der Spongiosa nach der 1 Woche. (Solcoseryl)

Es besteht ausgeprägter Callus im Canalis mandibulae und an der Corticalis, und zahlreiche neue Knochenpartikel in der Spongiosa. Es findet sich dichtes, regelmässiges Bindegewebe und zahlreiche neue Kapillaren in der Spongiosa.

2. Woche nach der Operation

Kontrolle (Abb. 6,7)

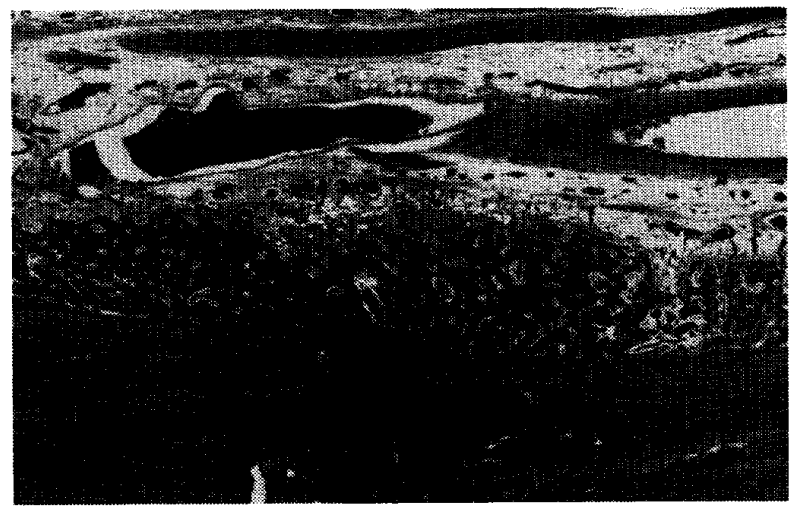

Abb. 6 Histologische Bild im Canalis mandibulae nach der 2 Woche. (Kontrolle)

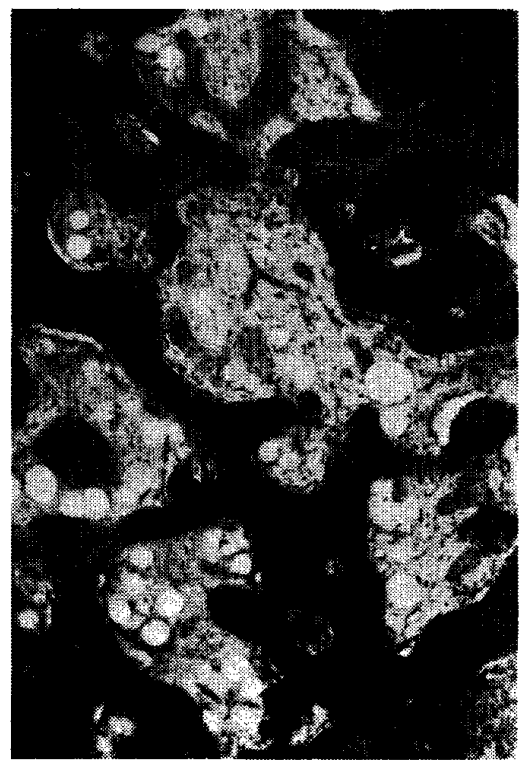

Abb. 7 Histologische Bild in der Spongios nach der 2 Woche. (Kontrolle) 
Es besteht Knochenanbau an der Frakturstelle und Knochenleiste im Canalis mandibulae. Es besteht viele Fettgewebe und wenig neue Knochensubstanz in der Spongiosa. Es findet sich wenig und locker Bindegewebe und Kapillarenneubildung in der Spongiosa.

Solcoseryl (Abb. 8,9)

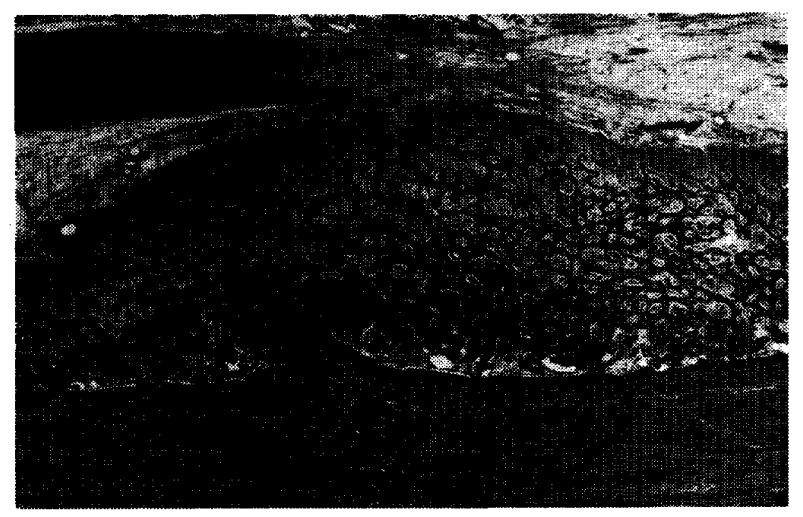

Abb. 8 Histologische Bild im Canalis mandibulae nach der 2 Woche. (Solcoseryl)

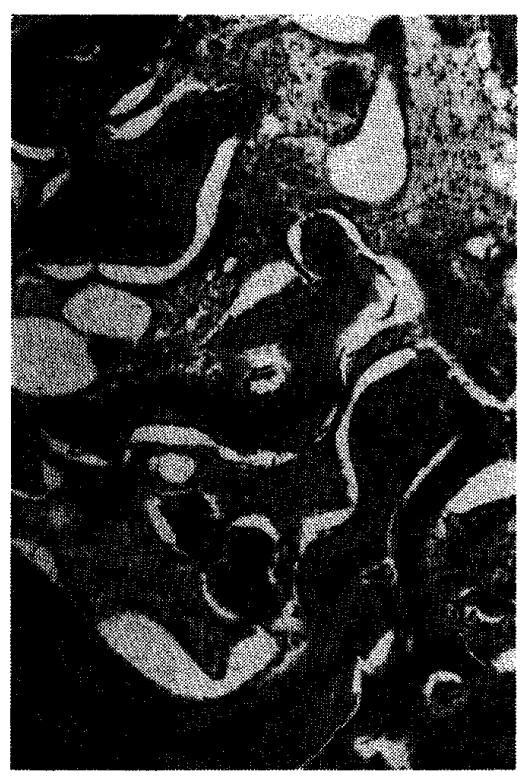

Abb. 9 Histologische Bild in der Spongiosa nach der 2 Woche. (Solcoseryl)

Es besteht starker Knochenanbau in der Corticalis und Füllung des Canalis mandibulae bis zum Nervus mandibularis. Es besteht wenig Fettgewebe, zahlreiche neue Knochensubstanz in der Spongiosa. Es findet sich dichtes Bindegewebe und grosse, neugebildete Gefässe in der Spongiosa.

3. Woche nach der Operation

Kontrolle (Abb. 10,11)

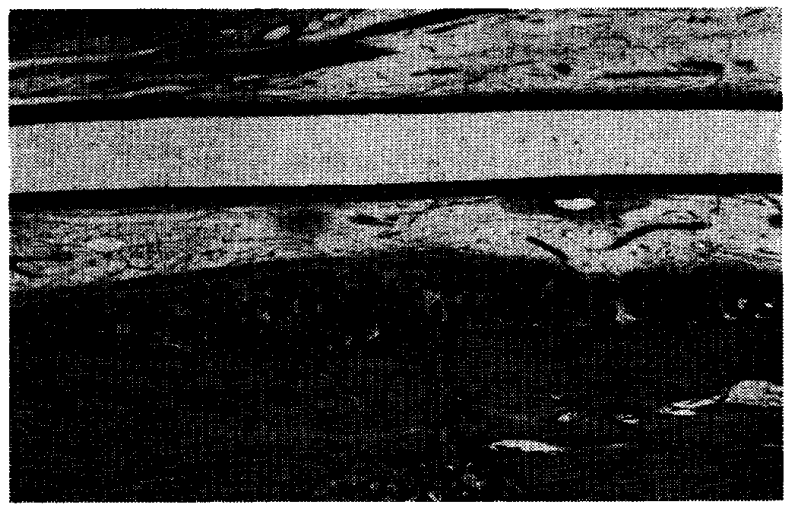

Abb.10 Histologische Bild im Canalis mandibulae nach der 3 Woche. (Kontrolle)

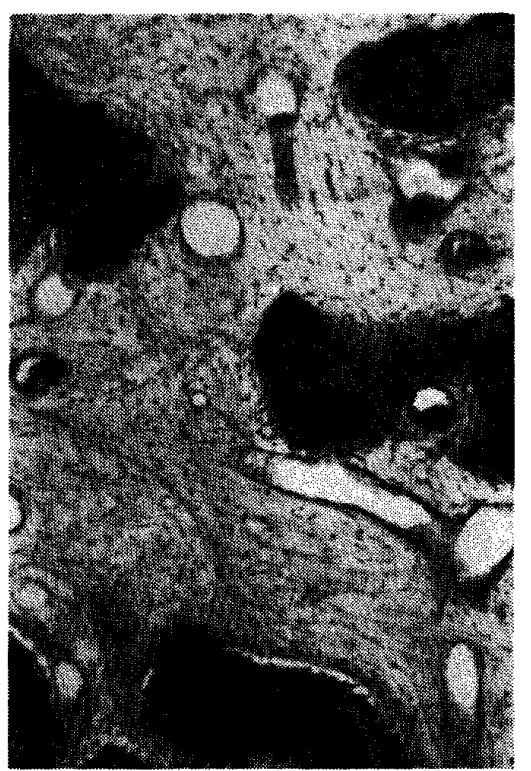

Abb.11 Histologische Bild in der Spongiosa nach der 3 Woche. (Kontrolle) 
Es besteht zahlreicher, lockerer Knochenanbau und Knochenneubildung im Frässpalt. Es besteht geringe Knochenneubildung, lockere Knochenmatrix und ausgeprägtes Fettgewebe in der Spongiosa. Es findet sich gut ausgebildet Bindegewebe und Kapillarenneubildung in der Spongiosa.

Solcoseryl (Abb. 12,13)

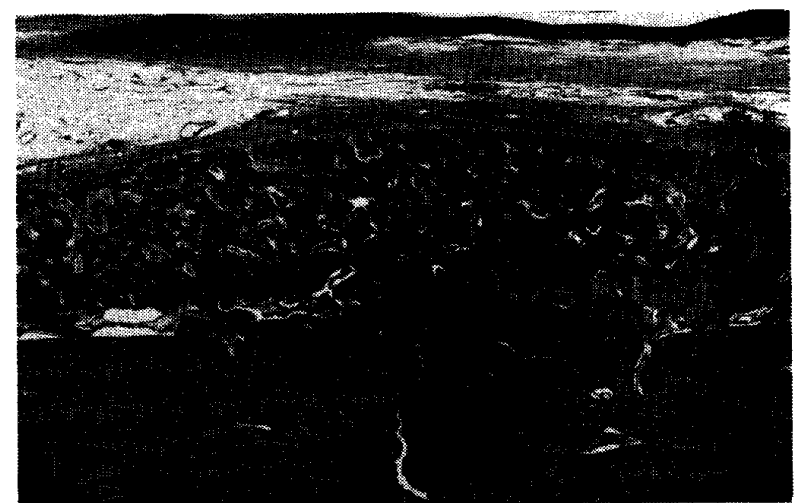

Abb.12 Histologische Bild im Canalis mandibulae nach der 3 Woche. (Solcoseryl)

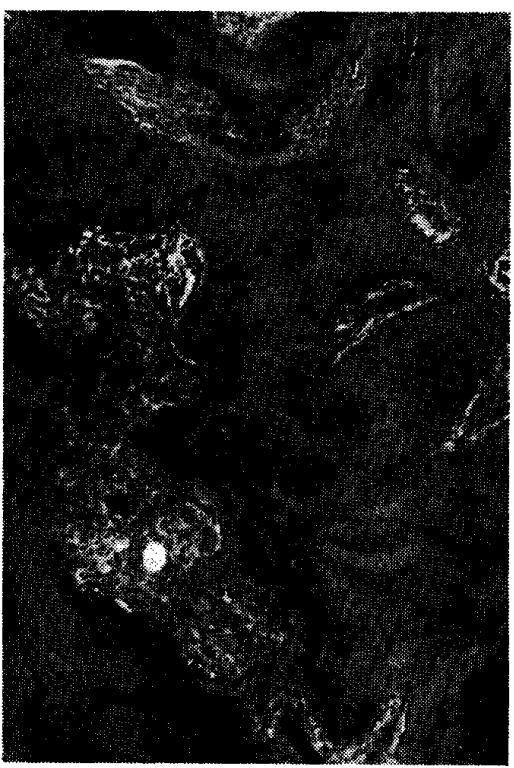

Abb.13 Histologische Bild in der Spongiosa nach der 3 Woche. (Solcoseryl)

Es besteht dichte Knochenmatrix mit Gefässversorgung am Colticalisrand. Es besteht aktive Knochenneubildung, dichte Knochenmatrix und wenig Fettgewebe in der Spongiosa. Es findet sich sehr gut ausgebildet Bindegewebe und dichtes neues Kapillarnetz in der Spongiosa.

\section{Diskussion}

Die Ergebnisse der mit Solcoseryl behandelten Hunde sind eindeutig besser als die der Kontrolltiere. Nach der ersten, zweiten und dritten Woche sind jeweils auffallende Unterschiede $z u$ beobachten: Die Callusbildung, der Einbau von Knochenbälkchen in den Corticalis, die Neubildung von Kapillaren und Knochensubstanz in der Spongiosa und der Aufbau von Bindegewebe in der Spongiosa sind bei den Solcoseryl-Tieren wesentlich stärker ausgeprägt.

Der Autor bewertet das Ergebnis folgendermassen: Bei allen Solcoseryl-Tieren ist die Reparation der Fraktur im Vergleich zur Kontrolle beschleunigt. Die Ergebnisse mit Solcoseryl nach der 2. Woche sind vergleichbar mit den unbehandelten Kontrolltieren nach der 3 . Woche.

Der Versuch zeigt, dass Solcoseryl die Reossifikation eines Bruches qualitativ in positiver Weise beeinflusst. 


\section{Zusammenfassung}

Ziel dieser orientierenden Studie ist es zu prüfen, ob Solcoseryl die Heilung von Knochenfrakturen qualitativ und quantitativ beeinflusst.

Der Versuch zeigt, dass Solcosryl die Reossifikation eines Bruches qualitativ in positiver Weise beeinflusst.

\section{Schrifttum}

1) Hayashida, T., Aoyagi, T., Itoh, R., Mizushima, N., Yamaga, S., Tsumura, S., Matsumoto, H. und Takahashi, R. : Kiso to Rinsho $8: 142-168$, 1974. (auf dem Japanische)

2) Kurokawa, T., Furukawa, K. und Sasaki, T. : Kiso to Rinsho 8: 169-176, 1974. (auf dem Japanische)

3 ) Irino, A., Miyoshi, I., Sezaki, T., Dairi, S. und Nagamori, G. : Shinyaku to Rinsho 13:7881, 1964. (auf dem Japanische)

4 ) Kawamata, K., Tachikawa, I., Takada, H., Watanabe, T. und Yuasa, S. : Shinyaku to Rinsho $14: 15-18,1965$. (auf dem Japanische)

5 ) Araki, G. : Kiso to Rinsho 8:200-206, 1974. (auf dem Japanische)

6) Mihara, H. : Kiso to Rinsho $12: 147-179$, 1978. (auf dem Japanische)

7) Spiessl, B. : New concepts in maxillofacial bone surgery. Springer-Verlag, Berlin, Heidelberg, New York, 1976, 27-34. 


\section{顎骨骨折に及ぼすソルコセリルの影響に関する}

\section{実験的研究 (1)}

福出蚱一

九州歯科大学口腔外科学第 1 講座（指導：山田長敬教授）

スイス, Solco Basel 社から発売されている Solcoseryl が贯, 十二指腸潰瘍, 腫瘍並びに脳血管障害の治 療剤として効果をあげているととは多くの文献報告で明 らかであり，日本では Solcoseryl 注射が使用されてい る、しかし，骨折や骨欠損に対する報告例は日本ではみ られず，またてれまで適応とされていない。

そこで著者は骨折の治療に Solcoseryl が定性的に， また定量的に影響を与えるか否か検索を行っている。
今回，6頭の 雑犬を 使用し，下顎骨骨体部を骨折さ せ，Solcoseryl 投与群と対象群を術後 $1,2,3$ 週に ついて病理組織学的に比較検索を加えた。

その結果，全ての Solcoseryl 群は骨折修復が対象群 に比較して早いてとがわかった。

このととから，Solcoseryl は骨折の際の骨再成に対 し，定性的には影響を及ぼすことが示唆された。 